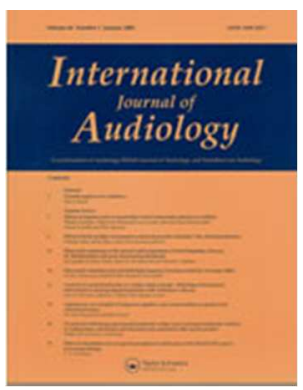

\title{
Uncomfortable Loudness Levels among children and adolescents seeking help for tinnitus and/or hyperacusis
}

\begin{tabular}{|r|l|}
\hline Journal: & International Journal of Audiology \\
\hline Manuscript ID & TIJA-2017-11-0354 \\
\hline Manuscript Type: & Original Paper \\
\hline Date Submitted by the Author: & 22-Nov-2017 \\
\hline Complete List of Authors: & $\begin{array}{l}\text { Aazh, Hashir; Royal Surrey County Hospital, Audiology; London School of } \\
\text { Hygiene \& Tropical Medicine, Department of Social and Environmental } \\
\text { Health Research } \\
\text { McFerran, Don; Essex County Hospital, Otolaryngology; } \\
\text { Moore, Brian; University of Cambridge, Dept of Experimental Psychology }\end{array}$ \\
\hline Keywords: & Tinnitus, Behavioral Measures, Pediatric, Noise \\
\hline \multicolumn{2}{|c}{} \\
\hline
\end{tabular}

SCHOLARONE $^{\text {IM }}$

Manuscripts 
Uncomfortable Loudness Levels among children and adolescents seeking help for tinnitus and/or hyperacusis

Hashir Aazh*, Don McFerrant \& Brian C. J. Moore ( $^{\ddagger}$

"Audiology Department, Royal Surrey County Hospital NHS Foundation Trust, Egerton

Road, Guildford, GU2 7XX, UK, †ENT Department, Colchester Hospital University NHS

Foundation Trust, Colchester CO4 5JL, UK, ${ }^{\ddagger}$ Department of Experimental Psychology,

University of Cambridge, Downing Street, Cambridge CB2 3EB, UK

Correspondence: Dr. Hashir Aazh, Tinnitus \& Hyperacusis Therapy Specialist Clinic, Audiology Department, Royal Surrey County Hospital, Egerton Road, Guildford, GU2 7XX, UK. E-mail: hashir.aazh@nhs.net 
Key words: tinnitus, hyperacusis, children, misophonia 
Aazh et al ULLs among children with tinnitus and/or hyperacusis

\title{
Uncomfortable Loudness Levels among children and adolescents seeking help for tinnitus and/or hyperacusis
}

\author{
Hashir Aazh*, Don McFerran ${ }^{\dagger} \&$ Brian C. J. Moore ${ }^{\ddagger}$ \\ *Audiology Department, Royal Surrey County Hospital NHS Foundation Trust, Egerton \\ Road, Guildford, GU2 7XX, UK, ${ }^{\dagger}$ ENT Department, Colchester Hospital University NHS \\ Foundation Trust, Colchester CO4 5JL, UK, ${ }^{\ddagger}$ Department of Experimental Psychology, \\ University of Cambridge, Downing Street, Cambridge CB2 3EB, UK
}

\section{Short title}

ULLs among children with tinnitus and/or hyperacusis

Key words: tinnitus, hyperacusis, children, misophonia

\author{
Abbreviations \\ BSA British Society of Audiology \\ PTA Pure tone average \\ SD Standard deviation \\ THTSC Tinnitus and Hyperacusis Therapy Specialist Clinic \\ ULL Uncomfortable Loudness Level \\ ULLmin Average ULL at $0.25,0.5,1,2,4$ and $8 \mathrm{kHz}$ for the ear with the lower \\ average ULL
}

Correspondence: Dr. Hashir Aazh, Tinnitus \& Hyperacusis Therapy Specialist Clinic, Audiology Department, Royal Surrey County Hospital, Egerton Road, Guildford, GU2 7XX, UK. E-mail: hashir.aazh@nhs.net 
Aazh et al ULLs among children with tinnitus and/or hyperacusis

\begin{abstract}
Objective: To assess the incidence of hyperacusis and severe hyperacusis among children and adolescents seen at an audiology outpatient tinnitus and hyperacusis service. Design: This was a retrospective study. Hyperacusis was considered as present if the average uncomfortable loudness level (ULL) at $0.25,0.5,1,2,4$ and $8 \mathrm{kHz}$ for the ear with the lower average ULL, which is denoted ULLmin, was $\leq 77 \mathrm{~dB}$ HL. Severe hyperacusis was considered as present if the ULL was $30 \mathrm{~dB}$ HL or less for at least one of the measured frequencies for at least one ear. Study sample: There were 62 young patients with an average age of 12 years ( $\mathrm{SD}=4.1$ years, range $4-18$ years). Results: $85 \%$ of patients had hyperacusis and $17 \%$ had severe hyperacusis. On average, ULLs at $8 \mathrm{kHz}$ were $9.3 \mathrm{~dB}$ lower than ULLs at $0.25 \mathrm{kHz}$. For $33 \%$ of patients, ULLs were at least $20 \mathrm{~dB}$ lower at 8 than at $0.25 \mathrm{kHz}$. Conclusions: Among children and adolescents seen at an audiology outpatient clinic for tinnitus and hyperacusis, hyperacusis diagnosed on the basis of ULLs is very prevalent and it is often characterized by lower ULLs at 8 than at $0.25 \mathrm{kHz}$.
\end{abstract}

Key words: tinnitus, hyperacusis, children, adolescents 
Tinnitus is the sensation of sound without any external acoustic sound source. Hyperacusis is intolerance of everyday sounds that causes significant distress and impairment in social, occupational, recreational, and other day-to-day activities (Aazh et al, 2016). The sounds may be perceived as uncomfortably loud, unpleasant, frightening, or painful (Tyler et al, 2014). There have been many studies of tinnitus and hyperacusis in adults, but relatively few studies in children. The need for further research on children with tinnitus and hyperacusis and the development of therapeutic services for such children has been highlighted by several authors (Coelho et al, 2007; Aazh et al, 2011; Baguley et al, 2013; Rosing et al, 2016a).

Estimates of the prevalence of tinnitus and hyperacusis in children vary from $4.7 \%$ to $46 \%$ for tinnitus and from 3.2\% to 17\% for hyperacusis (Coelho et al, 2007; Rosing et al, 2016b). Baguley et al (2013) combined data from clinics in several European countries that accepted referrals for tinnitus. They reported that among 87 children referred with a primary complaint of tinnitus, the severity of the tinnitus was classified as mild for $19(22 \%)$, moderate for $52(60 \%)$ and severe for $16(18 \%)$. They stated that the tinnitus was accompanied by hyperacusis in 34 cases (39\%), but they did not define the criteria used to diagnose hyperacusis.

Kentish et al (2000) reported that $46 \%(11 / 24)$ of children with tinnitus who were referred to a psychology department for tinnitus management complained about loud noises and the effects of the noises on their tinnitus. Such complaints could indicate the presence of hyperacusis, although this was not concluded explicitly by the authors. In a case review, Rosing et al (2016a) reported that 50\% (6/12) of children referred to audiology from EarNose-Throat departments and 28\% (19/69) of children referred to audiology from education and psychology settings for their tinnitus and/or hyperacusis were diagnosed with hyperacusis. They did not report the outcome of any audiological evaluations.

Uncomfortable Loudness Levels (ULLs) are often used to diagnose hyperacusis. For normal-hearing people, the average ULL is approximately $100 \mathrm{~dB}$ HL (Sherlock \& Formby, 2005). For people with hyperacusis, average ULLs are typically reduced (Tyler et al, 2014; Aazh \& Moore, 2017a). Coelho et al (2007) reported that 18\% of children (40 out of 222) aged 5-12 years who answered "yes" to the question "are you bothered by sounds?" had ULLs below $90 \mathrm{~dB}$ HL for at least at one frequency out of $0.25,0.5,1,2,3,4$, and $6 \mathrm{kHz}$ for at least one ear. Aazh and Moore (2017a) proposed diagnostic criteria for hyperacusis based on the average ULL across $0.25,0.5,1,2,4$ and $8 \mathrm{kHz}$ for the ear with the lower average ULL, which is denoted ULLmin. They suggested that a value of ULLmin equal to or below 
$77 \mathrm{~dB}$ HL should be taken as indicating the presence of hyperacusis (Aazh \& Moore, 2017a). With this ULLmin criterion, 95\% of adult patients diagnosed as having hyperacusis were found also to meet the criterion of a cut-off score on the Hyperacusis Questionnaire (HQ) (Khalfa et al, 2002) of 22 or more (Aazh \& Moore, 2017a). The proportion of children and adolescents seeking help for tinnitus and/or hyperacusis from audiology clinics who meet the ULLmin criterion for hyperacusis is not known.

Recently several authors have reported that some people with hyperacusis have ULLs as low as $30 \mathrm{~dB}$ HL (Sheldrake et al, 2015; Zaugg et al, 2016; Aazh \& Moore, 2017c). Aazh and Moore (2017c) proposed that severe hyperacusis should be diagnosed when a ULL of 30 $\mathrm{dB} H \mathrm{HL}$ or less is measured for at least one of frequency from $0.25,0.5,1,2,3,4,6$, and 8 $\mathrm{kHz}$, for at least one ear. Using this criterion, they found that $4 \%$ of adults (average age $=56$ years, $\mathrm{SD}=16$ years) attending a specialist clinic for treatment of tinnitus and/or hyperacusis had severe hyperacusis. It is important to explore the proportion of children and adolescents who meet the criterion for severe hyperacusis, since this can lead to very severe problems in everyday life. The average sound level of a whisper or the background noise in a quiet library is about $30 \mathrm{~dB}$ SPL (ASHA, 2015).

The aim of this study was to assess the proportion of children and adolescents seen at an audiology outpatient tinnitus and hyperacusis service who met the ULL criteria for hyperacusis (ULLmin $\leq 77 \mathrm{~dB} \mathrm{HL}$ ) and severe hyperacusis (ULL of $30 \mathrm{~dB}$ HL or less for at least one of the measured frequencies).

\section{Method}

\section{Study design and patients}

This was a retrospective study conducted at the Tinnitus and Hyperacusis Therapy Specialist Clinic (THTSC), Royal Surrey County Hospital, Guildford, UK. The data for all patients aged 18 years or below who attended the THTSC between January 2013 and December 2016 were included $(n=62)$. These are referred to for brevity as "young" patients. The total number of patients over 18 years old with a primary complaint of tinnitus and/or hyperacusis who attended the THTSC in that period was 1565.

Demographic data for the young patients and the outcomes of their most recent audiological investigations were imported from the records held at the Audiology department. Audiological measures are listed below.

(1) Audiometry 
All young patients had appropriate audiometric testing but in several cases this had been performed in the referring clinic. As the therapeutic intervention offered at the THTSC is based on cognitive therapy (Aazh \& Allott, 2016), it was felt unnecessary to repeat the audiometric testing in these cases. However, to ensure consistency of the quality of data, audiometric data are presented only if the testing was performed in-house. Pure-tone audiograms were obtained based on the procedure described by the British Society of Audiology (BSA, 2011a). The severity of hearing loss was categorized based on the values of the pure-tone average (PTA) across 0.25, 0.5, 1, 2 and $4 \mathrm{~Hz}$ : Mild (20 - $40 \mathrm{~dB} \mathrm{HL})$, Moderate (41 - $70 \mathrm{~dB}$ HL), Severe (71 - $95 \mathrm{~dB}$ HL) and Profound (> $95 \mathrm{~dB}$ HL).

(2) Uncomfortable loudness levels (ULLs)

ULLs were measured using the BSA recommended procedure (BSA, 2011b). ULLs were only measured after a detailed explanation of the procedure had been given and the patient had understood and agreed to the procedure. For 21 out of 62 young patients, ULLs were not measured at any frequency, either because the patient did not seem to understand the instructions or because the patient/parent did not consent to the test.

\section{Ethical approval}

The study was approved by the South-West Cornwall and Plymouth Research Ethics Committee and the Research and Development Department at the Royal Surrey County Hospital.

\section{Data analysis}

The data were anonymized prior to statistical analysis. Descriptive statistics, specifically means and standard deviations (SD), are reported. Wilcoxon rank-sum tests were used to compare means. The $p$-value required for statistical significance was set at $p<0.05$. STATA (version 13) data analysis and statistical software was used for the analyses (StataCorp, 2013). The analyses were restricted to patients for whom there were complete data on all variables required for a particular analysis. The number of patients included in each analysis (n) is reported.

\section{Results}

A total of 62 young patients were assessed. Their average age was 12 years (range 4 - 18 years, $\mathrm{SD}=4.1$ years). Fifty two percent $(32 / 62)$ were male. Twelve patients were referred because they had tinnitus with no sound intolerance complaint, 17 had sound intolerance 
Aazh et al ULLs among children with tinnitus and/or hyperacusis

complaints without tinnitus, and 33 had a combination of tinnitus and sound intolerance complaints.

The mean audiometric thresholds and ULLs (across all frequencies from 0.25 to 8 $\mathrm{kHz})$ are presented in Table 1. The mean PTA across ears was $13 \mathrm{~dB}$ HL $(\mathrm{SD}=14.5)(\mathrm{n}=54$; these 54 were the patients whose pure-tone audiometric thresholds were measured across all frequencies required for calculation of the PTA). Based on the PTA for the worse ear, $80 \%$ (43 out of 54) were classified as having no hearing loss, 13\% (7/54) had mild hearing loss, $5.6 \%(3 / 54)$ had moderate hearing loss, and 1.8\% (1/54) had severe hearing loss.

We were not able to calculate ULLmin values for all young patients, since ULLs were not measured for all frequencies needed to calculate ULLmin. Among those for whom ULLmin values could be calculated, the mean value of ULLmin was $64 \mathrm{~dB}$ HL ( $\mathrm{SD}=15, \mathrm{n}=$ $34)$. Based on the ULLmin values, $85 \%$ (29/34) of the young patients had hyperacusis.

The mean ULL across all young patients and ears was $9.3 \mathrm{~dB}(\mathrm{SD}=16 \mathrm{~dB})$ lower at 8 than at $0.25 \mathrm{kHz}$. For those diagnosed as having hyperacusis, the mean ULL was $11.2 \mathrm{~dB}$ $(\mathrm{SD}=16 \mathrm{~dB})$ lower at 8 than at $0.25 \mathrm{kHz}$, while for those without hyperacusis the mean ULL was $1.2 \mathrm{~dB}(\mathrm{SD}=11 \mathrm{~dB})$ higher at 8 than at $0.25 \mathrm{kHz}$. However, the difference between those with and without hyperacusis failed to reach significance $(p=0.11)$. Nevertheless, for $33 \%$ (11/31) of young patients, ULLs averaged across the two ears were $20 \mathrm{~dB}$ or more lower at 8 than at $0.25 \mathrm{kHz}$ and all of these 11 patients were diagnosed with hyperacusis. The largest difference was $42.5 \mathrm{~dB}$.

The PTA averaged across ears was 13.6 dB HL $(\mathrm{SD}=17)$ for the patients with hyperacusis and $17 \mathrm{~dB}$ HL $(\mathrm{SD}=12 \mathrm{~dB})$ for the patients without hyperacusis. The difference between groups was not significant $(p=0.23$ ). The mean audiometric threshold at $8 \mathrm{kHz}$, averaged across ears, was $0.1 \mathrm{~dB} H L(\mathrm{SD}=12.5 \mathrm{~dB})$ lower than the mean threshold at 0.25 $\mathrm{kHz}$ for patients diagnosed with hyperacusis, while it was $2.5 \mathrm{~dB}$ HL $(\mathrm{SD}=10 \mathrm{~dB})$ higher for those with no hyperacusis. The difference between the two groups was not significant ( $p=$ $0.44)$.

Out of 41 patients, $7(17 \%)$ were diagnosed with severe hyperacusis as indicated by a ULL of $30 \mathrm{~dB}$ HL or less for at least one of the measured frequencies for at least one ear. A ULL of $30 \mathrm{~dB}$ HL or below occurred most often at $8 \mathrm{kHz}$ (see Table 2).

For the patients with severe hyperacusis, the mean value of ULLmin was $44.4 \mathrm{~dB} \mathrm{HL}$ $(\mathrm{SD}=6.8)$, while it was $68.4 \mathrm{~dB} \mathrm{HL}(\mathrm{SD}=12)$ for the remainder of the population $(p=$ 0.0004). For the patients with severe hyperacusis, the lowest value of ULLmin was $37.5 \mathrm{~dB}$ $\mathrm{HL}$ and the lowest ULL at a single frequency was $15 \mathrm{~dB}$ HL, which was recorded at 6 and 8 
$\mathrm{kHz}$ for one patient. There were no significant difference in age and gender distribution between patients with severe hyperacusis and the remainder of the population $(p=0.098$ and $p=0.3$, respectively). The PTA averaged across ears was $11 \mathrm{~dB} \mathrm{HL}(\mathrm{SD}=7 \mathrm{~dB})$ for patients with severe hyperacusis and $15 \mathrm{~dB}$ HL $(\mathrm{SD}=17 \mathrm{~dB})$ for the remainder of the population $(p=$ 0.72). The mean audiometric thresholds across ears was $0.7 \mathrm{~dB} H L(\mathrm{SD}=13 \mathrm{~dB})$ lower at 8 than at $0.25 \mathrm{kHz}$ for the patients diagnosed with severe hyperacusis, while it was and $3.9 \mathrm{~dB}$ $(\mathrm{SD}=20 \mathrm{~dB})$ higher but not significantly for the remainder of the population $(p=0.57)$.

The patients with severe hyperacusis tended to show more across-frequency variation in ULLs than the remainder of the population. The mean ULL (averaged across the two ears) was $17.5 \mathrm{~dB}(\mathrm{SD}=16 \mathrm{~dB})$ lower at 8 than at $0.25 \mathrm{kHz}$ for the patients with severe hyperacusis, while it was only $7.5 \mathrm{~dB}(\mathrm{SD}=15 \mathrm{~dB})$ lower for the remainder of the population. However, the difference was not statistically significant $(p=0.31)$.

For the study population as whole, the absolute value of the difference in average ULLs across ears was $5.0 \mathrm{~dB}(\mathrm{SD}=5 \mathrm{~dB})$. The maximum difference in average ULL across ears was $17.5 \mathrm{~dB}$. The absolute value of the between-ear difference in average ULLs was 5.4 $\mathrm{dB}(\mathrm{SD}=4.7 \mathrm{~dB})$ for the patients with hyperacusis and $2.2 \mathrm{~dB}(\mathrm{SD}=3.2 \mathrm{~dB})$ for the reminder of the population $(p=0.076)$. The absolute value of the between-ear difference in average ULLs was $4.9 \mathrm{~dB}(\mathrm{SD}=4.8 \mathrm{~dB})$ for the patients with severe hyperacusis and $4.8 \mathrm{~dB}$ $(\mathrm{SD}=4.7 \mathrm{~dB})$ for the reminder of the population $(p=0.51)$. Thus, severe hyperacusis was not associated with greater interaural asymmetry.

\section{TABLES 1 AND 2 HERE}

\section{Discussion}

Limitations of the study

One limitation of this study was that complete data were not available for all of the young patients. This introduces a risk of selection bias (Pannucci \& Wilkins, 2010). 34\% of the young patients did not understand the instructions for ULLs or they or their parents did not consent to the procedure. The lack of consent probably reflects anxiety that the test might involve the presentation of sounds that would be perceived as uncomfortable or annoying. This highlights the need to modify the test instructions and procedure to make them more suitable for paediatric populations and to reduce the risk of discomfort, especially for those with hyperacusis. A similar need has been discussed for adults with tinnitus and/or hyperacusis (Aazh \& Moore, 2017b), both in relation to the BSA recommended procedure for 
measurement of ULLs (BSA, 2011b) and in relation to the procedure for conducting puretone audiometry (BSA, 2011a).

A second limitation of this study is that the outcomes apply to a specific cohort of young patients who sought help for tinnitus and/or hyperacusis. The outcomes may not be representative of all children with tinnitus and hyperacusis.

\section{Referral rate for young people with tinnitus and/or hyperacusis}

In this study, 62 young patients were seen for tinnitus and/or hyperacusis management in a 4year period. This represents about $4 \%$ of the overall clinical caseload at the THTSC. A survey of four specialist tinnitus clinics in Europe showed even lower caseload percentages of children seen in those clinics, with figures varying from $0.02 \%$ to $0.9 \%$ of the total clinical caseload (Baguley et al, 2013). Local awareness among those making referrals will affect how many children are referred. The low referral rate together with the high prevalence of tinnitus in the young population, as reviewed in the introduction, suggest that there is an unmet need and that education of those making referrals is needed.

\section{Audiometric characteristics of the sample}

The mean PTA across the frequencies 0.25 to $8 \mathrm{kHz}$ of the young people seeking help for tinnitus and/or hyperacusis was $13 \mathrm{~dB}$ HL $(\mathrm{SD}=14.5 \mathrm{~dB})$ which is lower than the mean PTA of $22 \mathrm{~dB}$ HL ( $\mathrm{SD}=16 \mathrm{~dB}$ ) reported for the adult population seeking help for tinnitus and/or hyperacusis at the same clinic (Aazh \& Moore, 2017a). The great majority (80\%) of the young population had PTA values within the normal range, indicating that tinnitus and hyperacusis often occur without any manifestation of hearing problems in the audiogram. Hyperacusis was not characterized by strong across-frequency variations in audiometric threshold or by large interaural asymmetry in PTA.

\section{Incidence of hyperacusis}

Based on the ULLmin values, $85 \%$ (29/34) of the young patients who sought help for their tinnitus and/or hyperacusis were diagnosed with hyperacusis. This is consistent with the reasons given for referral, since $81 \%(50 / 62)$ of the young patients were referred because of sound intolerance problems, either alone $(n=17)$ or in combination with tinnitus $(n=33)$. The incidence of hyperacusis in our sample is higher than the $39 \%$ incidence rate reported by Baguley et al (2013) for young patients, but their sample had a primary complaint of tinnitus, whereas our sample was based on young patients seeing help for tinnitus, hyperacusis, or 
both. The prevalence found in our study is also higher than the $36 \%(177 / 494)$ prevalence of hyperacusis reported using the same ULLmin criterion in a study of patients with tinnitus and/or hyperacusis with an average age of 55 years $(S D=17$ years) (Aazh \& Moore, 2017a).

Seventeen percent (7/41) of the young patients in the current study were diagnosed with severe hyperacusis, as indicated by ULLs of $30 \mathrm{~dB}$ HL or less for at least one of the measured frequencies for at least one ear. This proportion is considerably higher than the $4 \%$ prevalence of severe hyperacusis (13/362), among patients with an average age of 56 years $(\mathrm{SD}=16$ years), diagnosed using the same criterion (Aazh \& Moore, 2017c).

Overall, it appears that among patients seeking help for tinnitus and/or hyperacusis, tinnitus is more prevalent among older people, while hyperacusis is more prevalent among young people. This could partly be a consequence of the fact that the referral pathway for young people is not as established as that for adults, at least in the UK. As a result, only a small proportion of young patients are referred for tinnitus and/or hyperacusis rehabilitation. It is possible that only children with severe problems are being referred to specialist tinnitus and hyperacusis clinics, and that hyperacusis leads to more severe problems than tinnitus. This could lead to the relatively high prevalence rate for severe hyperacusis observed in our study. Future studies should explore this further.

\section{Characteristics of ULLs for young patients with hyperacusis}

The most common frequency associated with ULLs of $30 \mathrm{~dB}$ HL or below was $8 \mathrm{kHz}$. Averaged across ears with hyperacusis, the mean ULL at $8 \mathrm{kHz}$ was $11.2 \mathrm{~dB}$ lower than at $0.25 \mathrm{kHz}(\mathrm{SD}=16)$. This does not seem to be related to the difference in the corresponding audiometric thresholds, as the mean difference in audiometric thresholds between 8 and 0.25 $\mathrm{kHz}$ across the young patients diagnosed with hyperacusis was only $-0.1 \mathrm{~dB}$. Coelho et al (2007) reported that, for children with tinnitus and/or hyperacusis, the mean ULL at $8 \mathrm{kHz}$ was only about $4 \mathrm{~dB}$ lower than that at $0.25 \mathrm{kHz}$. However, a smaller proportion of their young patients had hyperacusis than was the case in our study. For $33 \%$ of our young patients $(11 / 31)$, the ULL at $8 \mathrm{kHz}$ was $20 \mathrm{~dB}$ or more lower than the ULL at $0.25 \mathrm{kHz}$. This proportion is higher than for patients with an average age of 55 years ( $\mathrm{SD}=17$ years) seen in the same clinic, of whom $23 \%$ had a $20 \mathrm{~dB}$ or more difference between ULLs at 8 and 0.25 $\mathrm{kHz}$ (Aazh \& Moore, 2017a). The mean difference in ULL thresholds between $8 \mathrm{kHz}$ and $0.25 \mathrm{kHz}$ across left and right ears for the young patients with severe hyperacusis was over twice that for the remainder of the sample. However this difference was not statistically 
significant, possibly because of the small sample size. Further research is needed to assess whether severe hyperacusis among young patients is associated with unusually high sensitivity to high-frequency sounds.

Studies of adults with hyperacusis have typically shown that ULLs averaged across participants did not change markedly across the frequency range (Formby et al, 2007; Meeus et al, 2010; Sheldrake et al, 2015; Aazh \& Moore, 2017a), although there was often a trend for ULLs to be lower at $8 \mathrm{kHz}$ than at middle frequencies, especially for patients with little or no hearing loss. Most of these studies did not report the variation of ULLs across frequency for individual patients. The strong across-frequency variation in ULLs for many of our young patients might be an indication of adverse reactions only to specific sounds, which is consistent with the definitions of annoyance and fear hyperacusis (Tyler et al, 2014) and misophonia (Cavanna \& Seri, 2015; Kumar et al, 2017). Future studies should explore the pattern of ULLs for individual young patients and their relationship to the everyday sounds that are found to be aversive by the patients.

\section{Conclusions}

Several studies have shown that tinnitus and hyperacusis are common in childhood. Yet few children are referred for treatment of their tinnitus and/or hyperacusis. In this study, which was conducted in a specialist clinic for the treatment of tinnitus and hyperacusis, only 62 children presented in a four-year period, representing $4 \%$ of the clinical caseload.

The results of this study suggest that there are some common characteristics among children and adolescents who are referred for help for their tinnitus and/or hyperacusis:

(1) Approximately $80 \%$ had pure tone audiograms within the normal range.

(2) More than $85 \%$ had hyperacusis, as diagnosed based on ULLmin values less than or equal to $77 \mathrm{~dB}$ HL.

(3) $17 \%$ had severe hyperacusis, as diagnosed based on a ULL of $30 \mathrm{~dB}$ HL or less for at least one of the measured frequencies for at least one ear.

(4) Strong across-frequency variations in ULL were common, ULLs typically being lower at 6 and $8 \mathrm{kHz}$ than at lower frequencies. For 33\% of young patients, the ULL was at least 20 $\mathrm{dB}$ lower at 8 than at $0.25 \mathrm{kHz}$; all of these patients were diagnosed with hyperacusis.

\section{Acknowledgements}

The authors would like to thank the staff at the Tinnitus and Hyperacusis Therapy Specialist Clinic at the Royal Surrey County Hospital for their help in data collection. 
Declaration of interests: The authors have no interests to declare.

\section{References}

Aazh, H. \& Allott, R. 2016. Cognitive behavioural therapy in management of hyperacusis: a narrative review and clinical implementation. Auditory and Vestibular Research, 25, 63-74.

Aazh, H. \& Moore, B.C.J. 2017a. Factors related to Uncomfortable Loudness Levels for patients seen in a tinnitus and hyperacusis clinic. International Journal of Audiology 56, 793-800.

Aazh, H. \& Moore, B.C.J. 2017b. Incidence of discomfort during pure-tone audiometry and measurement of uncomfortable loudness levels among People seeking help for tinnitus and/or hyperacusis American Journal of Audiolgy, 26, 226-232.

Aazh, H. \& Moore, B.C.J. 2017c. Prevalence and characteristics of patients with severe hyperacusis among patients seen in a tinnitus and hyperacusis clinic Journal of American Academy of Audiology, in press,

Aazh, H., Moore, B.C.J., Lammaing, K. \& Cropley, M. 2016. Tinnitus and hyperacusis therapy in a UK National Health Service audiology department: Patients' evaluations of the effectiveness of treatments. Int J Audiol, 55, 514-522.

Aazh, H., Moore, B.C.J. \& Prasher, D. 2011. Providing support to school children with hyperacusis. British Journal of School Nursing, 6, 174-178.

ASHA 2015. Noise. Audiology Information Series, 10802, 1-2.

Baguley, D.M., Bartnik, G., Kleinjung, T., Savastano, M. \& Hough, E.A. 2013. Troublesome tinnitus in childhood and adolescence: data from expert centres. Int J Pediatr Otorhinolaryngol, 77, 248-51.

BSA 2011a. Pure-tone air-conduction and bone-conduction threshold audiometry with and without masking: Recommended Procedure. Reading, UK: British Society of Audiology.

BSA 2011b. Recommended Procedure: Determination of uncomfortable loudness levels. Reading, UK: British Society of Audiology

Cavanna, A.E. \& Seri, S. 2015. Misophonia: current perspectives. Neuropsychiatr Dis Treat, 11, 211723.

Coelho, C.B., Sanchez, T.G. \& Tyler, R.S. 2007. Hyperacusis, sound annoyance, and loudness hypersensitivity in children. Prog Brain Res, 166, 169-78.

Formby, C., Gold, S.L., Keaser, M.L., Block, K.L. \& Hawley, M.L. 2007. Secondary benefits from tinnitus retraining therapy: clinically significant increases in loudness discomfort level and expansion of the auditory dynamic range. . Semin Hear, 28, 227-260.

Kentish, R.C., Crocker, S.R. \& McKenna, L. 2000. Children's experience of tinnitus: a preliminary survey of children presenting to a psychology department. Br J Audiol, 34, 335-40.

Khalfa, S., Dubal, S., Veuillet, E., Perez-Diaz, F., Jouvent, R. et al 2002. Psychometric normalization of a hyperacusis questionnaire. ORL J Otorhinolaryngol Relat Spec, 64, 436-42.

Kumar, S., Tansley-Hancock, O., Sedley, W., Winston, J.S., Callaghan, M.F. et al 2017. The Brain Basis for Misophonia. Current Biology, 1-7.

Meeus, O.M., Spaepen, M., Ridder, D.D. \& Heyning, P.H. 2010. Correlation between hyperacusis measurements in daily ENT practice. Int J Audiol, 49, 7-13.

Pannucci, C.J. \& Wilkins, E.G. 2010. Identifying and avoiding bias in research. Plast Reconstr Surg, $126,619-25$. 
Aazh et al ULLs among children with tinnitus and/or hyperacusis

Rosing, S.N., Kapandais, A., Schmidt, J.H. \& Baguley, D.M. 2016a. Demographic data, referral patterns and interventions used for children and adolescents with tinnitus and hyperacusis in Denmark. Int J Pediatr Otorhinolaryngol, 89, 112-20.

Rosing, S.N., Schmidt, J.H., Wedderkopp, N. \& Baguley, D.M. 2016b. Prevalence of tinnitus and hyperacusis in children and adolescents: a systematic review. BMJ Open, 6, e010596.

Sheldrake, J., Diehl, P.U. \& Schaette, R. 2015. Audiometric characteristics of hyperacusis patients. Front Neurol, 6, 105.

Sherlock, L.P. \& Formby, C. 2005. Estimates of loudness, loudness discomfort, and the auditory dynamic range: normative estimates, comparison of procedures, and test-retest reliability. $J$ Am Acad Audiol, 16, 85-100.

StataCorp 2013. Stata Statistical Software: Release 13. TX, USA: StataCorp LP.

Tyler, R.S., Pienkowski, M., Rojas Roncancio, E., Jun, H.J., Brozoski, T. et al 2014. A review of hyperacusis and future directions: part I. definitions and manifestations. Am J Audiol, 23, 402-419.

Zaugg, T.L., Thielman, E.J., Griest, S. \& Henry, J.A. 2016. Subjective Reports of Trouble Tolerating Sound in Daily Life versus Loudness Discomfort Levels. Am J Audiol, 25, 359-363. 
Table 1. Means (SDs) of the pure-tone audiometric thresholds and uncomfortable loudness levels (ULLs) in dB HL, shown separately for the right and left ears. The value of $n$ indicates the number of patients for whom a given measure was obtained.

\begin{tabular}{lccccccccccc}
\hline \multicolumn{10}{c}{ Frequency, $k H z$} \\
\hline 10
\end{tabular}


Table 2. Number of patients with a ULL of $30 \mathrm{~dB}$ HL or less at each frequency $(0.25,0.5,1$, $2,3,4,6$, and $8 \mathrm{kHz})$, shown separately for the right and left ears $(\mathrm{n}=41)$.

\begin{tabular}{lllllllll}
\hline & \multicolumn{1}{c}{ Frequency, $\mathrm{kHz}$} \\
\hline & 0.25 & 0.5 & 1 & 2 & 3 & 4 & 6 & 8 \\
\hline Right ear & 0 & 0 & 0 & 2 & 0 & 0 & 2 & 3 \\
Left ear & 1 & 0 & 0 & 1 & 1 & 1 & 1 & 3 \\
\hline
\end{tabular}

